\title{
Evaluations of computational techniques for the engraving of projectiles
}

\author{
J. South, B. Powers \& M. Minnicino \\ US Army Research Laboratory, Aberdeen Proving Grounds, MD, USA
}

\begin{abstract}
This paper presents research on the evaluations of computational methods used to model the engraving of small caliber projectiles. The launch mechanics of a $5.56 \mathrm{~mm}$ projectile fired from a rifled barrel based on a M16A2 are examined in this study. The interaction between small caliber projectiles and rifled barrels is evaluated using finite element analysis (FEA) simulations utilizing the 3D explicit dynamic FEA LS-DYNA code. The calculations were performed to understand the interaction of the projectile during the initial portion of launch, where the projectile is subjected to the dynamic pressure from the combustion of the charge. The modeling parameters investigated include the order of element integration, the hourglass control scheme, and contact algorithms. The stress and strain state within both the projectile and the barrel are evaluated and compared to experimental data. The results show that a comparison of global hourglass energy to internal energy can lead to improper conclusions about the validity of the engraving model. The choice of the hourglass scheme and contact algorithm affects the hourglass energy and may affect the internal energy. This effort has led to increased insight into the behavior of the engraving process on small caliber projectiles and the numerical techniques necessary to reduce modeling difficulties.

Keywords: engraving, projectile, hourglass.
\end{abstract}

\section{Introduction}

Correct simulation of the engraving process is crucial for the design of obturation and prediction of in-bore dynamics. In addition, given realistic constitutive models, consideration of the utility of different materials for the bullet can be made. Unfortunately the engraving process is a difficult 
phenomenon to model. During the engraving process excessive element deformations often lead to erroneous results. A possible solution is to utilize element erosion; however, this approach assumes that the elements and the corresponding volume of material engraving is worn away, which may not capture the effect of material yielding and flow around the rifling of a barrel during the launch of a projectile. Two dimensional modeling can be applied $[1,2]$ to evaluate the axi-symmetric interaction of a projectile with a barrel but this approach precludes a true rifling profile with lands and grooves. Full three dimension modeling is required to properly evaluate the engraving process.

In this study evaluations of the computational techniques for the engraving of $5.56 \mathrm{~mm}$ projectiles were performed. The interaction between small caliber projectiles and rifled barrels was evaluated using finite element analysis (FEA) simulations utilizing the 3D explicit dynamic FEA code LS-DYNA. A simplifying assumption in these analyses was that the rifling profile was without twist and that the interactions could be assessed with a quarter symmetry model. Excessive element deformations and high hourglass energies were present in the initial modeling efforts and caused numerical difficulties. In an effort to address these issues, a series of modifications were implemented into the models to evaluate the effects of the hourglass control scheme, order of integration of the elements, and the contact algorithm,

\section{Model development}

Finite element models (FEM) were developed of a $5.56 \mathrm{~mm}$ projectile fired from a rifled barrel based on a M16A2. As previously stated a simplifying assumption in these analyses was that the rifling profile was without twist. This assumption provided the ability to generate quarter symmetric models. The standard projectile evaluated in the models was the M855 projectile. This projectile contains a hardened steel penetrator backed by a lead-antimony slug encased in a copper gilding full metal jacket body [3].

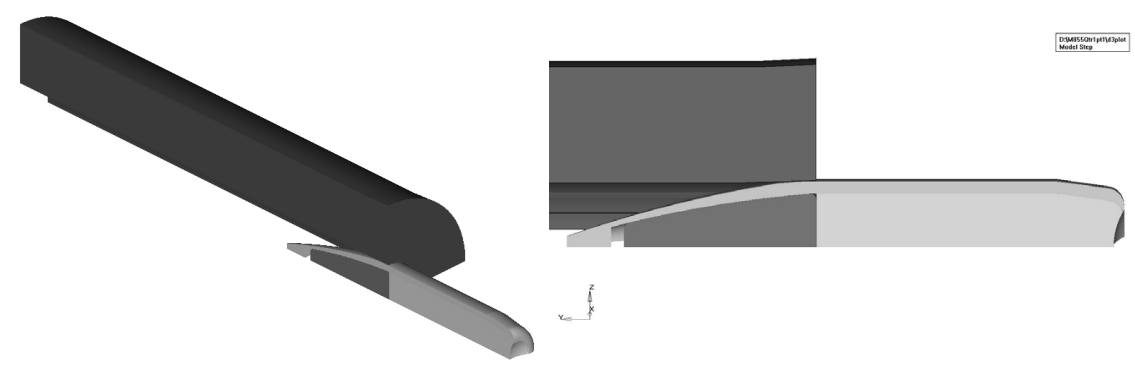

Figure 1: Graphics of the quarter symmetry model showing two different views.

The geometry for both the projectile and the weapon [4] were obtained from their respective technical drawing packages. The barrel was assumed to be an ideal barrel with perfect symmetry and centerline, and a greatly reduced length to 
decrease computation time since only initial engraving was to be studied. Axial boundary conditions were applied to the base of the barrel to prevent its motion. The base pressure-time curve for the M855 was obtained from the interior ballistic code IBHVG2.

The simulations were performed using LS-DYNA [5]. Contact was used between the slug-core-jacket and the jacket-barrel. All of the components within the projectile were kinematically constrained by contact surfaces. The material properties for the components in the model were obtained from experimental data. A graphic of the model is show in Figure 1. The baseline model possesses six elements thru the thickness of the copper gilding metal and thirty six elements across the ninety degree sweep of the quarter symmetry. The baseline contact algorithm was surface to surface with the master and slave pairs identified by parts. The baseline hourglass control was the stiffness form.

\subsection{Quasi-static model of push test}

A quasi-static model was used to explore dynamic effects by comparing with the baseline model. While the material properties defined in the model are not rate dependent, inertial and transient effects are still present in the baseline model. Also, the results of quasi-static experiments are available where a projectile was slowly pushed through a barrel [6]. In these experiments an M855 projectile was statically pushed thru an M16A2 barrel at a given displacement rate. The load, displacement of the push rod, and hoop strain on the outer diameter of the barrel were recorded in a manner similar to that described in [7]. This data allows for a validation of the model with the experiments.

The geometry of the baseline dynamic model was used for the quasi-static analysis. Mass scaling was used to artificially increase the density, in turn increasing the time step through the Courant Condition [5]. This allows quasistatic simulations to be completed with an explicit finite element code using a minimum of computational time. The purpose of the "long time" simulation is to remove any transient and inertial effects from the simulation. The time step and termination time were arbitrarily chosen to be $5.0 \times 10^{-5} \mathrm{~s}$ and $2.5 \mathrm{~s}$, respectively. The time scale of the pressure-time history was linearly scaled to cover the entire defined run time of the model. Mass damping was applied to all materials in the model to damp out any oscillations in the solution due to solver noise. A value of $10 \%$ of critical damping at $5 \mathrm{~Hz}$ was used.

\subsection{Hourglass control and contact algorithm effects models}

The simulations to evaluate the affect of altering the contact algorithm also evaluated the incorporation of selective reduced fully integrated elements and viscous hourglass control. In these models the projectile was altered so that the internal components of the projectile were steel. This was chosen since previous work $[6,8]$ has shown that the choice of slug material affects the radial stress exerted on the rifling lands and the grooves. Steel was chosen so as to exacerbate this effect as a worst case scenario. 
The contact algorithm and element integration order and their corresponding model are presented in Table 1. The automatic option for contact in LS-DYNA changes how the surface normals of the search algorithm on master surface are calculated. The default option uses normals calculated at the nodes, where the automatic option calculates the normal on element edge segments. The fully integrated elements were only selected for the jacket and the lands and grooves. The remainder of the barrel was set at single point integration elements. The application of the fully integrated elements was chosen to compare the inherent elimination of hourglass modes with the increased computation time.

Table 1: $\quad$ Listing of the corresponding model and contact algorithm.

\begin{tabular}{|c|c|}
\hline Model & LS-Dyna Contact Algorithm \\
\hline A & Surface to Surface \\
\hline B & Automatic Surface to Surface \\
\hline C & $\begin{array}{c}\text { Surface to Surface with Fully Integrated Elements } \\
\text { Viscous Hourglass Control }\end{array}$ \\
\hline D & $\begin{array}{c}\text { Surface to Surface with Fully Integrated Elements and } \\
\text { Elements }\end{array}$ \\
\hline E & Automatic Surface to Surface with Fully Integrated \\
\hline
\end{tabular}

\section{Model results}

\subsection{Quasi-static model of push test}

The quasi-static models were post processed to compare the hoop strain to the experimental data and to evaluate the selection of model control parameters. To verify that the simulation is quasi-static, the kinetic energy was verified to be negligible compared to the internal energy. The hourglass energy was examined to verify that the energy preventing the hourglass modes was negligible to the internal energy. The ratio of the hourglass energy to the internal energy was found to be a maximum of $2.0 \%$ after engraving started.

The predicted strain data was compared between the static and dynamic cases at a location $1.27 \mathrm{~cm}$ from the chamber end of the barrel section. Figure 2 shows the predicted hoop strain and the average experimental hoop strain plotted against projectile displacement. For comparison, the strains could not be plotted versus time since the projectiles in the model and experiment would not be at the same location for a given time. The average experimental hoop strain from the push test has a maximum of approximately $105 \mu \varepsilon$. The maximum predicted hoop strain from the quasi-static model is $170 \mu \varepsilon$. The longitudinal shift between the two data sets is due to the fact that the strain gauges were placed further down the barrel in the experimental setup. This increased distance was the result of experimental constraints within the test fixture. The maximum dynamic strain was approximately 1.5 times greater than the quasi-static strain. 


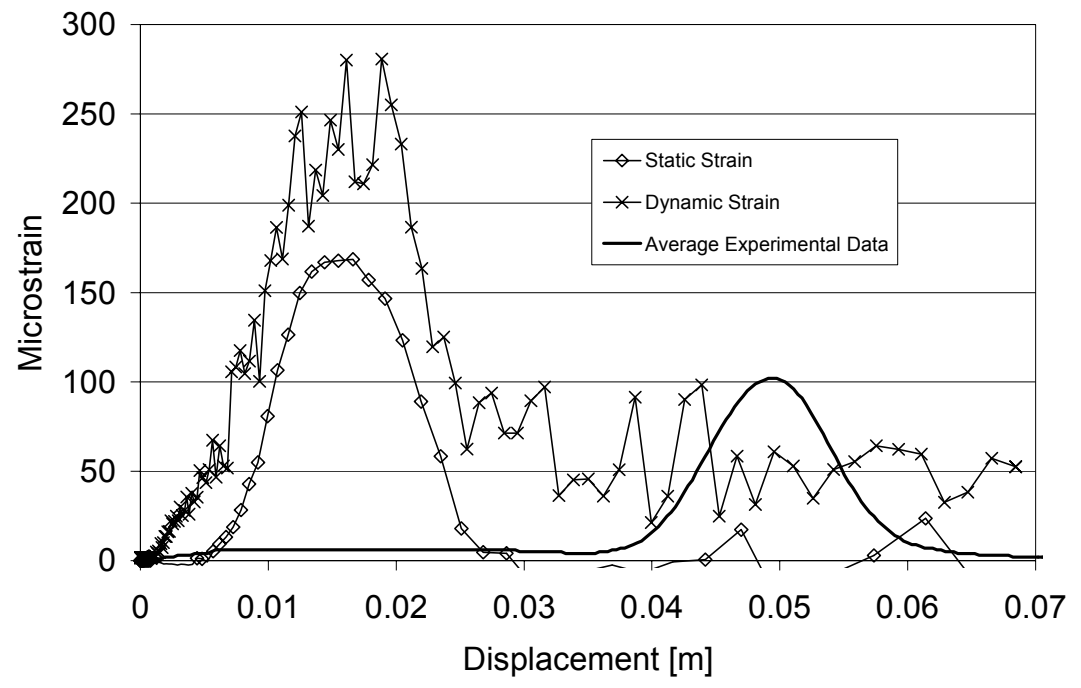

Figure 2: $\quad$ Plot of the predicted strain for the static and dynamic simulations as well as experimental strain from a push test.

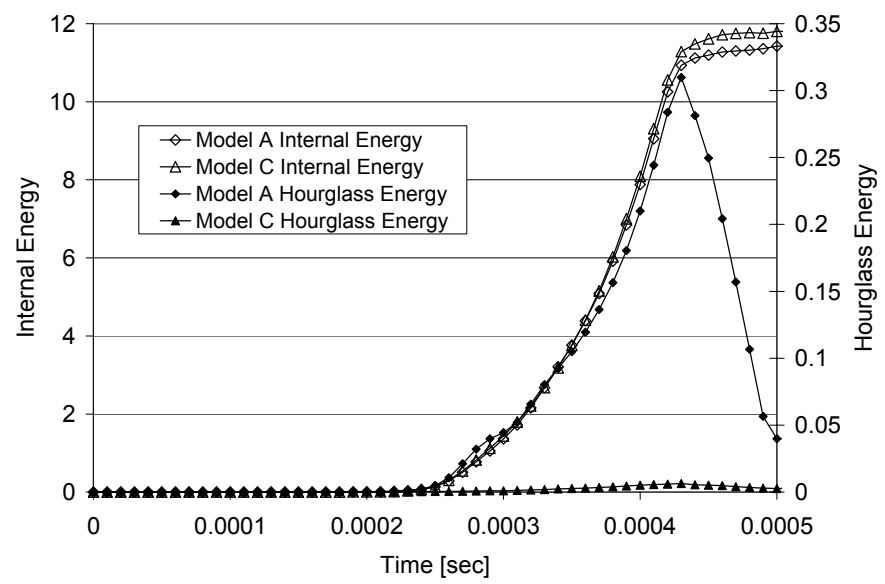

Figure 3: Plot showing internal (open symbols) and hourglass (filled symbols) energy.

\subsection{Contact algorithm effects}

The affect of the contact algorithm, element and material selections was quantified by comparison of the internal energy and hourglass energy. Figure 3 shows the representative internal and hourglass energies for models A and C. 
The $0.25 \mathrm{~ms}$ delay in the appearance of measurable energy is due to the time necessary to build sufficient pressure to begin engraving of the projectile. The hourglass energies in the figure begin to increase after $0.25 \mathrm{~ms}$ and then decrease as the time approaches $5 \mathrm{~ms}$. The decrease is the result of the projectile exiting the barrel section.

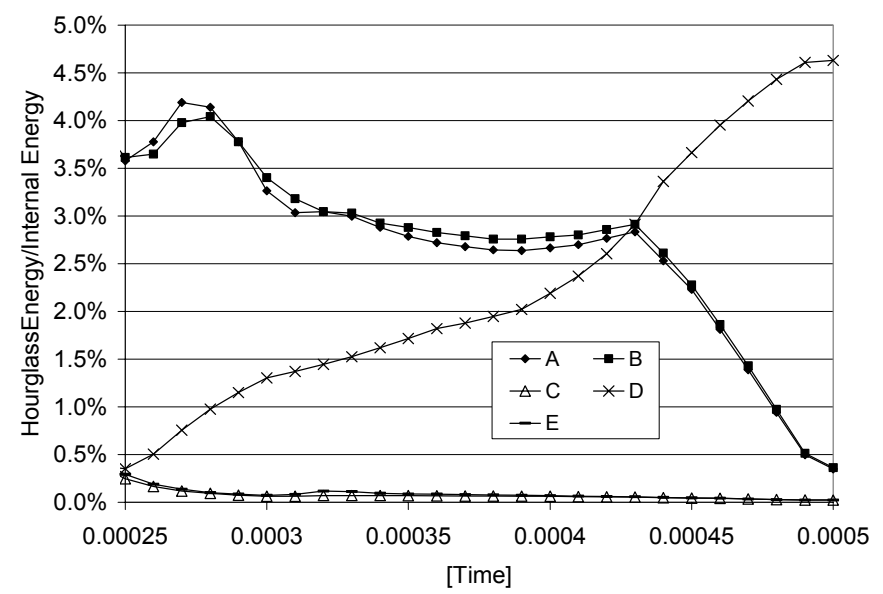

Figure 4: Plot of the ratio of hourglass energy to internal energy for the six models.

The ratio of the hourglass energy to the internal energy was plotted for the five models. Figure 4 shows these ratios as a function of time. The data in the figure between zero and $0.25 \mathrm{~ms}$ are not valid as the data is the quotient of two small numbers. Figure 3 shows that the internal and hourglass energies are very small between zero and $0.25 \mathrm{~ms}$. Once engraving begins the ratios begin to consolidate into groups. Models $\mathrm{A}$ and $\mathrm{B}$, without higher integration order elements, yielded a ratio with a maximum of $4 \%$. Models $\mathrm{C}$ and $\mathrm{E}$ with some fully integrated elements yielded a maximum ratio of less that $0.5 \%$. There was an average $40 \%$ increase in the run time between models with and without the fully integrated elements. Model D with the viscous hourglass control demonstrated an increasing ratio during the time that the projectile was engraved. This value peaked at approximately $4.5 \%$. All of the contact formulations with stiffness hourglass controls improved with time.

\subsection{Local effect of hourglass control algorithm}

As shown previously, the hourglass control algorithm has a large effect on the global hourglass energy. The engraving process has large local stresses, which might drive local hourglassing. This local effect might not be seen in the global energy comparisons. For this application, the state of stress in the land is of particular interest, so the effect of the hourglass control algorithm on the land stress is compared. 
The radial stress on a land in the first element forward of the forcing cone is shown in Figure 5. All three cases show an initial peak when the projectile first contacts the element, and then a sharp change in stress level. The Stiffness Control case shows a tensile radial stress after the sharp change in stress level, and then the stress sharply oscillates. The radial stress in the Viscous Control case stays compressive and oscillates around an average value. The Fully Integrated case has the smoothest time history. The radial stress stays compressive, and the magnitude of the oscillations is the lowest of the three cases.

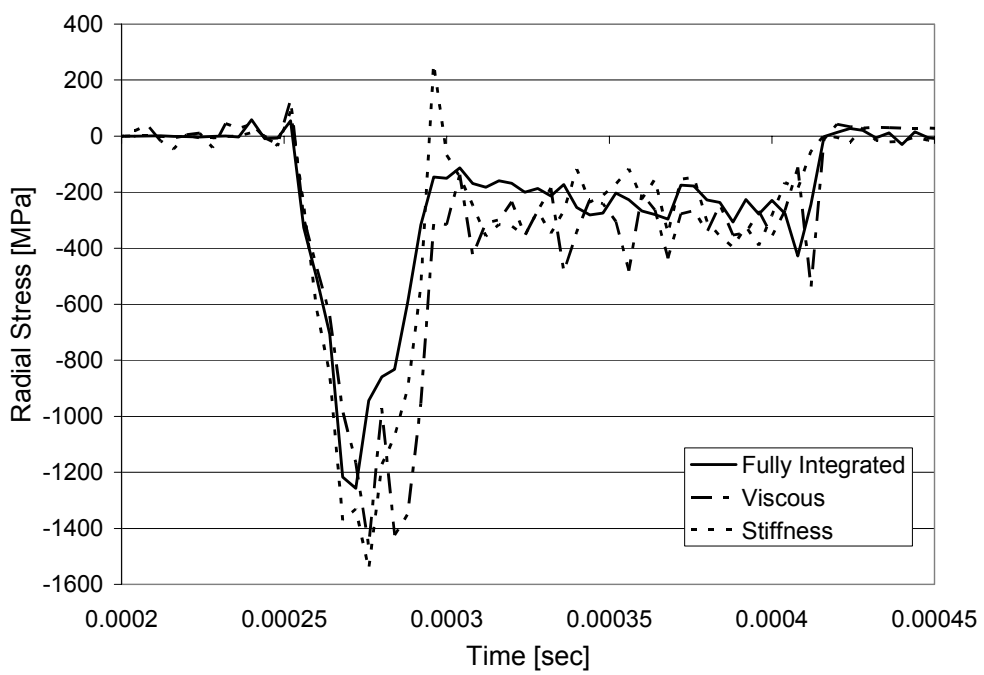

Figure 5: The radial stress in a land as a function of time for the Fully Integrated, Viscous Control, and Stiffness Control.

\section{Discussion}

\subsection{Quasi-static model}

The quasi-static model acts as a bridge between the dynamic model and the experimental results. The comparison of the maximum hoop strain shows reasonable agreement between the quasi-static model and the experimental data. The strain was recorded over a land in the quasi-static FE model where the maximum affect of the passing projectile would be expected. This difference may be because the experiment uses a real barrel where the lands have a twist, which might cause the strain measurement taken at fixed location on the barrel to decrease faster as the projectile moves by. Also, the FE analysis only modeled a section of the barrel $3.8 \mathrm{~cm}$ in length and the projectile is approximately $2.3 \mathrm{~cm}$ long. The shorter barrel length is more compliant since the end faces are not constrained, allowing the faces to move radially. Therefore, the model might deform more as the projectile passes, than the experimental barrel. 
The agreement between maximum strains from the experiment and quasistatic model gives confidence that assumptions made in creating the FE models are reasonable. The dynamic strain is expected to be greater because of inertial effects that are likely to results in projectile dilation. This is seen in the results for the dynamic FE model, where the maximum is approximately 1.5 times greater than the quasi-static model.

\subsection{Global energy effects}

The simulations to evaluate the effect of the contact algorithm, element integration order and hourglass control yielded clear data. The affect of including the automatic option onto surface to surface, models A and B and C and E, had very little effect on the ratio of the hourglass to the internal energy. There was some reduction in the ratio by use of this option but the reduction was on the order of $0.1 \%$. There was no change to the solution time of the model. As expected the use of fully integrated elements for portions of the model greatly reduced the overall hourglass energy and thus the ratio. This decrease was a factor of eight as the ratio was reduced from $4 \%$ to $0.5 \%$; however, this decrease was offset by a $40 \%$ increase in run time. For more complex models, a substantially longer runtime could be a factor in meeting design timeframe constraints.

\subsection{Local effects}

One advantage of the finite element method is the ability to inspect local effects. While the stiffness hourglass control is shown to be more effective than the viscous algorithm from a global energy perspective, care must be taken when validating the model. In general, viscous hourglass control is preferred for simulations involving high rates of deformation. Stiffness hourglass control should be used when the rates of deformation are low.

When investigating the radial stress in a land, the stiffness control gave the least reliable results, even calculating a tensile radial stress when this is physically impossible. This is most likely due to the element hourglassing, making the stress prediction incorrect. In a large model such as this, a small number of elements hourglassing will not affect a global energy comparison. But if the primary region of interest is near those elements, the model cannot be used for quantitative analysis.

The viscous control algorithm predicted a much more realistic time history for the radial stress. This is not surprising since the strain rates at the contact interface are expected to be rather high. The rest of the model, which includes the majority of the barrel, the core, and penetrator, experiences low strain rates. Therefore the viscous control has minimal effect on suppressing the hourglassing in large sections of the model. A global energy comparison highlights this problem and shows the stiffness control performing better at suppressing hourglassing, even though the viscous control predicts a more realistic stress in the land. 
The use of fully integrated elements in the land prevents the hourglassing in the area of interest and predicts a realistic radial stress history. The computational cost of fully integrated elements is high, which is why they were only used locally. Another drawback is that fully integrated elements cannot undergo large deformations like reduced integration elements. Used sparingly, fully integrated can be used to insure trustworthy results in areas of importance.

\section{Conclusions}

In this study, evaluations were conducted to investigate different computational techniques for the engraving of a $5.56 \mathrm{~mm}$ projectile fired from a rifled barrel based on a M16A2. The results show the choice of the contact algorithm, element order of integration, and hourglass control can drastically affect the accuracy of the model as well as the run time. The baseline model was evaluated quasi-statically and validated against experimental data. The choice in contact algorithm can affect the hourglass energy. For this engraving model the use of a stiffness form of hourglass control yielded the best results from a global energy perspective, while the viscous formulation appeared to result in energy ratios that were increasing. However, the local stress in the land shows that the viscous control predicts more realistic stress history. The partial use of fully integrated elements provided the expected reduction in the energy ratio however this reduction, from $4 \%$ to $0.5 \%$, was at the expense of a $40 \%$ increase in run time.

The results of this modeling effort have led to increased insight into the behavior of the engraving process on small caliber projectiles and the numerical techniques necessary to reduce numerical difficulties. The models should be tailored to the problem being solved to provide the most accurate results. Future work will include combinations of hourglass and viscous hourglass control in different parts of the engraving model in an effort to save on the computational cost of using fully integrated elements.

\section{References}

[1] Chen P.C.T., "Analysis of Engraving and Wear in a Projectile Rotating Band," U.S. Armament Research, Development and Engineering Center Technical Report ARCCB-TR-99012, Watervliet, NY, USA, July 1999

[2] Chen P.C.T., "Modeling of Barrel/Projectile Interaction in a Rotating Band," U.S. Armament Research, Development and Engineering Center Technical Report ARCCB-TR-01011, Watervliet, NY, USA, June 2001

[3] M855 Technical Drawing Package, Armament Research, Development and Engineering Center Picatinny Arsenal, NJ, USA, 1980

[4] M4A1 Technical Drawing Package, Armment Research, Development and Engineering Center, Rock Island, IL, 2001

[5] LS-DYNA Users Manual, Livermore Software Technology Corporation, Livermore, CA, USA, 2003 
[6] South J., Yiournas A., Minnicino M., "The Effect of Slug Material on the Behavior of Small Caliber Ammunition," U.S. Army Research Laboratory Technical Report ARL-TR-3901, APG, MD, USA, 2006

[7] Siewert J., "Small Caliber Engraving Force Measurements," Presented at the NDIA Small Arms Systems Section Annual Symposium, Exhibition and Firing Demonstration, Las Vegas, NV, USA, 10-13 May 2004, www.ndia.org

[8] South J., Newill J., Kamdar D., Middleton J., Hanzl F., DeRosa G., "Bridging the Gap Between the Art and Science of Materials for Small Caliber Ammunition," Amptiac Quarterly, 8(4), pp. 57-63, 2004. 